Kröll, Martin

\title{
Das Zusammenspiel von Selbst- und Fremdorganisation in lernenden
}

\section{Organisationen}

Faßhauer, Uwe [Hrsg.]; Fürstenau, Bärbel [Hrsg.]; Wuttke, Eveline [Hrsg.]: Jahrbuch der berufs- und wirtschaftspädagogischen Forschung 2013. Opladen [u.a.] : Verlag Barbara Budrich 2013, S. $245-257$. (Schriftenreihe der Sektion Berufs- und Wirtschaftspädagogik der Deutschen Gesellschaft für Erziehungswissenschaft (DGfE))

口院运回 Quellenangabe/ Reference:

Kröll, Martin: Das Zusammenspiel von Selbst- und Fremdorganisation in lernenden Organisationen - In: Faßhauer, Uwe [Hrsg.]; Fürstenau, Bärbel [Hrsg.]; Wuttke, Eveline [Hrsg.]: Jahrbuch der berufsund wirtschaftspädagogischen Forschung 2013. Opladen [u.a.] : Verlag Barbara Budrich 2013, S. 245-257 - URN: urn:nbn:de:0111-opus-80792 - DOI: 10.25656/01:8079

https://nbn-resolving.org/urn:nbn:de:0111-opus-80792 https://doi.org/10.25656/01:8079

in Kooperation mit / in cooperation with:

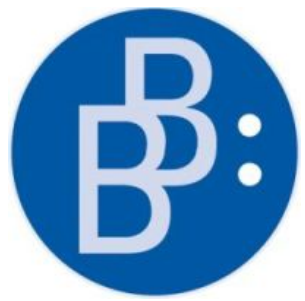

https://www.budrich.de

\section{Nutzungsbedingungen}

Dieses Dokument steht unter folgender Creative Commons-Lizenz: http://creativecommons.org/licenses/by-nc-nd/3.0/de/deed - Sie dürfen das Werk bzw. den Inhalt unter folgenden Bedingungen vervielfältigen, verbreiten und öffentlich zugänglich machen: Sie müssen den Namen des Autors/Rechteinhabers in der von ihm festgelegten Weise nennen. Dieses Werk bzw. dieser Inhalt darf nicht für kommerzielle Zwecke verwendet werden und es darf nicht bearbeitet, abgewandelt oder in anderer Weise verändert werden.

Mit der Verwendung dieses Dokuments erkennen Sie die Nutzungsbedingungen an.

\section{Terms of use}

This document is published under following Creative Commons-License: http://creativecommons.org/licenses/by-nc-nd/3.0/de/deed.en - You may copy, distribute and transmit, adapt or exhibit the work in the public as long as you attribute the work in the manner specified by the author or licensor. You are not allowed to make commercial use of the work or its contents. You are not allowed to alter, transform, or change this work in any other way.

By using this particular document, you accept the above-stated conditions of use.

\section{Kontakt / Contact:}

\section{peDOCS}

DIPF | Leibniz-Institut für Bildungsforschung und Bildungsinformation Informationszentrum (IZ) Bildung

E-Mail: pedocs@dipf.de

Internet: www.pedocs.de

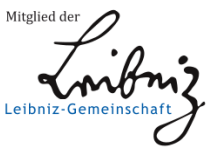




\section{Jahrbuch der berufs- und wirtschaftspädagogischen Forschung 2013}

Uwe Faßhauer, Bärbel Fürstenau, Eveline Wuttke (Hrsg.)

DGfE Deutsche Gesellschaft 
Jahrbuch der berufs- und wirtschaftspädagogischen Forschung 2013 
Schriftenreihe der Sektion

Berufs- und Wirtschaftspädagogik der Deutschen Gesellschaft

für Erziehungswissenschaft (DGfE) 
Uwe Faßhauer

Bärbel Fürstenau

Eveline Wuttke (Hrsg.)

Jahrbuch der berufs- und

wirtschaftspädagogischen

Forschung 2013

Verlag Barbara Budrich

Opladen • Berlin • Toronto 2013 
Bibliografische Information der Deutschen Nationalbibliothek

Die Deutsche Nationalbibliothek verzeichnet diese Publikation in der Deutschen Nationalbibliografie; detaillierte bibliografische Daten sind im Internet über http://dnb.d-nb.de abrufbar.

(C) Dieses Werk ist im Verlag Barbara Budrich erschienen und steht unter folgender Creative Commons Lizenz: http://creativecommons.org/licenses/by-nc-nd/3.0/de/ Verbreitung, Speicherung und Vervielfältigung erlaubt, kommerzielle Nutzung und Veränderung nur mit Genehmigung des Verlags Barbara Budrich.

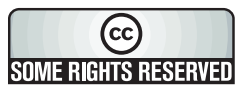

Dieses Buch steht im OpenAccess Bereich der Verlagsseite zum kostenlosen Download bereit (http://dx.doi.org/10.3224/978384740127)

Eine kostenpflichtige Druckversion (Printing on Demand) kann über den Verlag bezogen werden. Die Seitenzahlen in der Druck- und Onlineversion sind identisch.

\section{ISBN 978-3-8474-0127-8 \\ DOI $\quad 10.3224 / 978384740127$}

Umschlaggestaltung: bettina lehfeldt graphic design, Kleinmachnow Typografisches Lektorat: Ulrike Weingärtner, Gründau Verlag Barbara Budrich, http://www.budrich-verlag.de 


\title{
Teil I: Lehr-Lernforschung in der beruflichen Bildung
}

\author{
Jan Hendrik Stork \\ Der Einfluss unterschiedlicher Unterrichtsmodelle auf den Erwerb \\ von mathematischen und kaufmännischen Kompetenzen im
} beruflichen Unterricht

Mandy Hommel

„Darf ich um Ihre Aufmerksamkeit bitten?!” Wege aus dem

Aufmerksamkeitstief....

Stephan Schumann, Franz Eberle, Maren Oepke

Ökonomisches Wissen und Können am Ende der Sekundarstufe II:

Effekte der Bildungsgang-, Klassen- und Geschlechtszugehörigkeit

Christine Caroline Jähnig

Assessing Business Knowledge of Students in German

Higher Education

Anja Mindnich, Stefanie Berger, Sabine Fritsch

Modellierung des fachlichen und fachdidaktischen Wissens von

Lehrkräften im Rechnungswesen - Überlegungen zur Konstruktion

eines Testinstruments.

Roland Happ, Susanne Schmidt, Olga Zlatkin-Troitschanskaia

Der Stand des wirtschaftswissenschaftlichen Fachwissens von

Bachelorabsolventen der Universität und der Fachhochschule.

\section{Teil II: Didaktik und Methodik beruflichen Lernens}

Nicole Kimmelmann, Wilhelmine Berg

Wie viel Deutsch darf's sein? Das Projekt „Deutsch am Arbeitsplatz”

Claudia Stolp, Jens Siemon

Wirkung auf Lernerfolg und Motivation durch Debriefing in

Unternehmensplanspielen . 99 
Georg Tafner

Supranationalität begreifbar machen. Performative Pädagogik

im Planspiel.

Frank Arens

Praxisbegleitung in der Pflegeausbildung - ein blinder Fleck der

Berufsbildungsforschung?

Axel Grimm

Lehrerhandeln im computerunterstützten Berufsschulunterricht Handlungsmuster von Berufsschullehrkräften in elektro- und metalltechnischen Lehr-Lernarrangements

\section{Teil III: Hochschuldidaktik und Lehrerbildung}

\section{Gabriela Kugler}

Grundstrukturen professionellen Lehrerwissens: Wissen zur

Lesekompetenzförderung von Berufsschullehrkräften

Juliana Schlicht

Forschendes Lernen im Studium: Ein Ansatz zur Verknüpfung von

Forschungs-, Lehr- und Lernprozessen.

Romy Wolff

Interaktionsprozesse zur Entscheidungsfindung in virtuellen und face-to-face Gruppen

Ulrike Weyland, Karin Reiber

Lehrer/-innen-Bildung für die berufliche Fachrichtung Pflege in hochschuldidaktischer Perspektive

\section{Teil IV: Berufs- und wirtschaftspädagogische Perspektiven}

Wiebke Petersen

Prinzipien der Regulierung des Feldes der Berufsbildung - ein Ansatz zum internationalen Vergleich von „ValNIL” 203 
Stefan Wolf

Berufsbildung und Migration - kritische Anmerkungen über

vernachlässigte Perspektiven

Markus Linten, Christian Woll

Berufsbildungsdiskurs 2010 und 2011: Vergleichende Resonanzanalyse referierter und nicht-referierter Zeitschriftenbeiträge zur

Berufsbildungsforschung und -praxis

Martin Kröll

Das Zusammenspiel von Selbst- und Fremdorganisation in lernenden Organisationen

Gregor Thurnherr, Samuel Schönenberger \& Christian Brühwiler

Hilfreiche Unterstützung in der Berufsorientierung aus Sicht von

Jugendlichen 


\title{
Das Zusammenspiel von Selbst- und Fremdorganisation in lernenden Organisationen
}

\author{
Martin Kröll
}

\section{Hintergrund und Fragestellung}

Die sich kontinuierlich wandelnden Herausforderungen der modernen Arbeitswelt führen zur Notwendigkeit von fortlaufender Mitarbeiterweiterbildung und lebenslangem Lernen. Um diesen Anforderungen gerecht zu werden, sind entsprechende Kompetenzen auf individueller und organisatorischer Ebene erforderlich. Es stellt sich damit die Frage, wie die entsprechenden Kompetenzentwicklungsaktivitäten organisiert werden und wie Lernprozesse für Mitarbeiter/ -innen motivierend, effizient und effektiv gestaltet werden können. Einhergehend mit dem Trend zum „Empowerment” von Organisationsmitgliedern, also der zunehmenden Gewährung von Entscheidungs- und Gestaltungsspielräumen im Arbeitsalltag, wird vorgeschlagen, auch das Lernen in Organisationen weitestgehend der Selbstorganisation (SO) der Individuen zu übertragen.

\subsection{Selbst- und Fremdorganisation von Lernen}

Der Begriff der Selbstorganisation des Lernens (SDL) bezieht sich darauf, inwieweit die betroffenen Akteure Ziele und Richtung der Kompetenzentwicklung, die Maßnahmen zur Umsetzung, den Zeitpunkt und den Transfer der erworbenen Kompetenzen sowie die Kriterien, nach denen sie den Erfolg der Kompetenzentwicklung evaluieren, selbst bestimmen können. Der Begriff der SDL ist vom Begriff der Selbststeuerung des Lernens abzugrenzen (vgl. Sembill/Seifried 2006, S. 94). Selbststeuerung liegt schon dann vor, wenn der Lernende einzelne Komponenten oder die Methode des Lernens selbst steuern kann, Ziele und Rahmen des Lernens jedoch von außen vorgegeben werden. Die Thematik der SDL wird seit mehreren Jahrzehnten aus bildungspolitischer und lerntheoretischer Sicht kontrovers diskutiert und wurde u.a. in Verbindung zu neuen Profilen beruflichen Verhaltens gesetzt (Baethge et al., 2003, S. 10). In diesem Artikel wird das Konstrukt der SDL in Abgrenzung von der Fremdorganisation (FO) des Lernens thematisiert und damit die weitreichende Organisation des Lernens durch Individuen gemeint.

Die Fremdorganisation von Lernen bezieht sich auf die von außen veranlassten bzw. gesteuerten Teile der Kompetenzentwicklung. Die FO kann bei- 
spielsweise durch die jeweiligen Führungskräfte, Kollegen, Personalentwicklungsabteilung, Unternehmensleitung, externe Weiterbildungsanbieter, Lieferanten (z.B. Herstellerschulung) oder andere Dritte beeinflusst werden. Im Fokus der Auseinandersetzung mit FO von Lernen in Organisationen steht bisher häufig die Rolle der Personal- bzw. Personalentwicklungsabteilung. Sie soll Inhalte und Form der Weiterbildung festlegen, die Mitarbeiter entsprechend unterstützen und ein lernförderliches Klima in der Organisation schaffen. Demgegenüber wurden die Rolle der Führungskräfte und die Bedeutung verschiedenen Führungsverhaltens in diesem Kontext bisher kaum untersucht. An dieses Forschungsdefizit knüpft der vorliegende Artikel an.

Im Folgenden wird FO primär über das kompetenzentwicklungsbezogene Verhalten der Führungskraft definiert, das heißt dass FO sich auf das Ausmaß bezieht, in dem Führungskräfte die Kompetenzentwicklung ihrer Mitarbeiter/ -innen unterstützen, steuern sowie kontrollieren. Dieser Fokus wird damit begründet, dass den Führungskräften im Hinblick auf die berufliche Kompetenzentwicklung eine zentrale Rolle zufällt. Die Führungskräfte legen häufig die Rahmenbedingungen fest, unter denen berufliche Kompetenzentwicklung stattfindet und/oder nehmen eine indirekte Vermittlerrolle ein. Selbst in dem Fall, dass die Personal- bzw. Personalentwicklungsabteilungen die Weiterbildungsprogramme organisieren und/oder Mitarbeiter/-innen selbst Kompetenzentwicklungsmaßnahmen vorschlagen, entscheiden die Führungskräfte über die Freistellung der Mitarbeiter/-innen zu Weiterbildungszwecken und die Unterstützung der Kompetenzentwicklung. Das Engagement der Führungskräfte in Bezug auf die Weiterbildung der Mitarbeiter/-innen schlägt sich letztendlich in der organisationalen Lernkultur der jeweiligen Organisation nieder (zur Diskussion der Kultur als gemeinsame Standards bzw. soziale Praktiken vgl. Reckwitz 2003). Weiterverstärkt wird die wichtige Rolle der Führungskräfte bei der Kompetenzentwicklung ihrer Mitarbeiter/-innen durch die Tendenz zur Dezentralisierung der Personalentwicklung, d. h. dass Personalentwicklung immer weniger zentral vorgegeben und organisiert wird, sondern auf der Meso- (d.h. Abteilungs-) und Mikroebene (d.h. einzelne Mitarbeiter/-innen) stattfindet. Diese Entwicklung führt dazu, dass die Bedeutung der Führungskräfte als Personalentwickler zunimmt und der Personalentwicklungsabteilung eine neue Rolle zufällt.

In der wissenschaftlichen Auseinandersetzung wird auf die Vorteilhaftigkeit der Nutzung von SDL am Arbeitsplatz hingewiesen (Park 2008). Die Förderung der SO des Lernens erfolgt dabei nicht primär aus humanistischen Gründen (wie z.B. Beitrag zur Persönlichkeitsentwicklung), sondern dient der Erzielung effektiverer Lernprozesse, die letztlich zu einer höheren Wettbewerbsfähigkeit der Organisation führen.

Es wird oftmals davon ausgegangen, dass die SDL in Kontrast zu fremdorganisierter Weiterbildung zu besseren Lernerfolgen führt. Dies wird damit begründet, dass Individuen ihre Bedürfnisse sowie Kompetenzpotentiale und 
-defizite selbst am besten kennen und somit die Relevanz des Gelernten für die jeweilige Arbeitswelt sichergestellt wird (Artis/Harris 2007). Auch die Diskussion um das „Theorem des Arbeitskraftunternehmers” (Voß/Pongratz 1998) weist auf die zunehmende Bedeutung der SO von Lernen in der heutigen Arbeitswelt hin. Gemäß diesem Theorem sind die Arbeitnehmer im zunehmenden Maße herausgefordert ihre Arbeitskraft selbst zu ökonomisieren, $\mathrm{zu}$ rationalisieren und zu kontrollieren sowie auf dem internen bzw. externen Arbeitsmarkt anzubieten. Das kontinuierliche Streben nach Selbstoptimierung im unternehmerischen Sinne ist von Organisationen durchaus gewünscht, da diese auf Unternehmer im Unternehmen („Intrapreneure”) angewiesen sind, um Innovationen zu generieren und ihre Wettbewerbsfähigkeit zu erhalten. Zudem tragen ein hoher Innovationsdruck, Dezentralisierungsprozesse sowie das Auftreten neuer Karrieremodelle zur Herausbildung eines Idealbildes vom proaktiven, selbstverantwortlichen und selbstorganisierten Arbeitnehmer bei.

Vor dem Hintergrund dieser Entwicklungen wird SO von Lernen als Lösung für einen großen Teil organisationaler Probleme vorgeschlagen. Die überaus positive Darstellung legt nahe, SDL als Managementtrend zu bezeichnen, der zum Teil unreflektiert auf verschiedenste Szenarien angewandt wird. In der SDL wird nicht zuletzt eine Möglichkeit gesehen, der zunehmenden Unsicherheit, mit denen Organisationen und Erwerbstätige sich konfrontiert fühlen, gerecht zu werden. Dabei wird allerdings oftmals vernachlässigt, dass SDL die Motivation und die Fähigkeit zur SO bei den Mitarbeitern/-innen voraussetzt. Nach Artis und Harris (2007) hängt die Bereitschaft zur SDL von verschiedenen Faktoren ab: (1) Persönliche Eigenschaft des Lernenden zur Selbstbestimmung, (2) Zutrauen im Hinblick auf die Fähigkeit, das Lernen selbst zu organisieren, (3) Kontextuelles Verständnis und Motivation zum Lernen sowie (4) Stabilität vs. Turbulenz der Lernumgebung und (5) Klima des organisatorischen Lernens. Je nach persönlicher Voraussetzung und Erfahrung des Lernenden können also verschiedene Grade der Unterstützung im Lernprozess notwendig sein, was auf die hohe Bedeutung der individuellen Abstimmung von FO und SO des Lernens auf den Arbeitnehmer hinweist.

\subsection{Das Zusammenspiel von Selbst- und Fremdorganisation}

Wenn unterschiedliche Anleitungsbedürfnisse im Lernprozess durch die Mitarbeiter/-innen bestehen, kann davon ausgegangen werden, dass sich einige Organisationsmitglieder mit der Übertragung der Verantwortung für ihre Kompetenzentwicklung überfordert fühlen, während andere sich noch mehr Freiraum in ihren Entscheidungen bezogen auf ihre Kompetenzentwicklung wünschen. Im vorliegenden Artikel wird angenommen, dass die Berücksich- 
tigung des Bedürfnisgrads nach FO bzw. SO in den meisten Organisationen nur unzureichend einbezogen wird. Mögliche nachteilige Folgen einer undifferenzierten Forderung der SDL in Organisationen wurden jedoch in der bisherigen Forschung kaum untersucht bzw. weitestgehend ignoriert.

An dieser Stelle stellt sich zudem die Frage, welches Führungsverhalten sich als vorteilhaft erweist, wenn es darum geht, die Organisation der Kompetenzentwicklung aus Sicht der Mitarbeiter/-innen zu unterstützen. Ein erster Schritt zur Auseinandersetzung mit dieser Problematik ist die Differenzierung aus der Führungsforschung zwischen mitarbeiter- und aufgabenorientiertem Führungsverhalten. Dabei beschreibt mitarbeiterorientiertes Führungsverhalten einen partizipativen Führungsstil, der durch offene, wechselseitige Kommunikation gekennzeichnet ist. Bei aufgabenorientierter Führung liegt der Fokus dagegen auf der Strukturierung der Arbeitsinhalte und Zuordnung von Aufgaben zu Mitarbeitern/-innen (Rowold/Heinitz 2008).

In diesem Zusammenhang ist zunächst die dichotome Messung von FO und SO auf einer Skala kritisch zu betrachten. Gemäß diesem Verständnis wird die Form des Lernens auf einem Kontinuum zwischen hoher SO und hoher FO eingeordnet. Dabei wird von der Annahme ausgegangen, dass das Ausmaß der SO mit steigender FO sinkt und umgekehrt. Wird nur auf den Lernprozess fokussiert, erscheint diese Auffassung verständlich. Wenn jedoch die Interaktion zwischen verschiedenen Akteuren, die an der Kompetenzentwicklung beteiligt sind, in die Analyse mit einbezogen wird, dann erscheint dieses Verständnis $\mathrm{zu}$ restriktiv. In Bezug auf die möglichen Kombinationen von SO und FO soll in diesem Artikel auf vier verschiedene Kategorien zurückgegriffen werden: 1. Die Weiterbildung der Mitarbeiter/innen kann beispielsweise in Organisationen zu einem hohen Maß institutionalisiert und damit fremdorganisiert sein, den Mitarbeitern/-innen aber trotzdem einen hohen Gestaltungsraum für Kompetenzentwicklung lassen (=hohe FO/hohe SO). 2. Ein anderer Fall liegt vor, wenn von den Organisationmitgliedern ein hohes Maß an SO im Hinblick auf ihre Kompetenzentwicklung gefordert wird, aber eine Unterstützung von dritter Seite nicht vorgesehen ist (= SO hoch, FO niedrig). Dies kann von Seiten der Organisation damit begründet werden, dass es aufgrund der zunehmenden Dynamik und Komplexität des Wirtschafts- und Arbeitslebens immer schwieriger wird, vorausschauend die Kompetenzentwicklungsbedarfe ihrer Organisationsmitglieder zu bestimmen und die zutreffenden Weiterbildungsmaßnahmen und inhalte auszuwählen. 3. Allerdings kann auch der umgekehrte Fall vorliegen (=FO hoch, SO niedrig): Die Organisation legt enorm viel Wert auf die Kompetenzentwicklung ihrer Organisationsmitglieder und gibt beispielsweise vor, welche Weiterbildungsmaßnahmen die Mitglieder besuchen und welche Kompetenzen sie erwerben sollen. Auf diese Weise soll sichergestellt werden, dass die Organisationsmitglieder über ein Mindestmaß an Kompetenzen verfügen. $\mathrm{Zu}$ viel Eigeninitiative wird als eher unerwünscht angese- 
hen. Die betreffenden Organisationsmitglieder sollten sich primär an die Vorgaben halten, die ihre Organisation an sie richtet, und es wird nicht von ihnen erwartet, dass sie weitgehend selbstständig Weiterbildungsziele und -methoden definieren. 4. Im letzten Fall sind sowohl SO als auch FO niedrig.

Ausgehend von den verschiedenen Kombinationsmöglichkeiten von SO und FO ist der Erkenntnis Rechnung zu tragen, dass Lernen auf verschiedenen Ebenen stattfindet kann. In diesem Zusammenhang wird häufig zwischen der individuellen Ebene, der Teamebene und der organisationalen Ebene differenziert. Um ein Mindestmaß an Effektivität und Effizienz der betrieblichen Kompetenzentwicklung zu erreichen, ist es sinnvoll diese Aktivitäten aufeinander abzustimmen. Einen Ansatzpunkt, um Lernen auf den unterschiedlichen Ebenen gerecht zu werden, stellt das theoretische Konzept um die Forschungsgruppe von Watkins dar (Watkins/Marsick 2003; Yang/ Watkins/Marsick 2004). Während die Beziehung zwischen den Ebenen und der Lernkultur in der bisherigen Forschung empirisch umfassend analysiert wurde, lag der Fokus bis jetzt nicht auf dem Einbezug von FO und SO des Lernens. Die zentrale forschungsleitende Fragestellung ist demnach wie folgt zusammenzufassen:

Wie wirken sich unterschiedliche Kombinationen von SO und FO des Lernens auf die Wahrnehmung der Lernkultur in Organisationen aus?

Der Begriff der Lernkultur wird je nach theoretischem Blickwinkel unterschiedlich definiert und operationalisiert. In der vorliegenden Arbeit soll Lernkultur aufbauend auf dem integrativen Konzept der lernenden Organisation nach Watkins und Marsick (2003) untersucht werden. Die Autoren gehen davon aus, dass Organisationen kontinuierlich lernen und sich dabei selbst transformieren. Dieser Prozess basiert auf dem Wechselspiel zwischen Organisationsmitgliedern und Organisationsstruktur, welche sich in einem interaktiven Prozess gegenseitig beeinflussen, und wird als zentraler Wettbewerbsvorteil eines Unternehmens in Zeiten strategischer Herausforderungen definiert (Yang/Watkins/Marsick 2004).

Watkins und Marsick (2003) entwickelten in empirischen Untersuchungen die nachfolgend genannten acht Dimensionen der lernenden Organisation, welche durch den von ihnen entwickelten DLOQ (Dimensions of the Learning Organization Questionnaire) erhoben werden können: (1) Kontinuierliches Lernen, (2) Zusammenarbeit und Lernen im Team, (3) Methoden der Erfassung von Lernen, (4) Empowerment, (5) Verbindungsstrukturen der Organisation, (6) Förderung strategischer Führung, (7) Ermöglichung von Rückfragen und Dialog, (8) Wissensleistung. Insgesamt umfasst der Fragebogen 62 Items. Aus den acht Skalen lässt sich nach Watkins und Marsick (2003) ein Gesamtindex bilden, welcher die Ausprägung der Lernkultur der jeweiligen Organisation beschreibt. Von den acht Dimensionen sind die ersten beiden auf individueller Ebene verortet, die dritte auf dem Team- oder 
Gruppenlevel und die übrigen auf organisationaler Ebene (Watkins/Marsick 2003).

\section{Hypothesen}

Wie bereits erläutert, wird in der Lernkulturforschung darauf hingewiesen, dass trotz oder gerade wegen des zentralen Stellenwerts der SO den Führungskräften eine wachsende, nicht zu ersetzende Aufgabenstellung zufällt. Demnach sollten die acht Dimensionen des DLOQ (Marsick/Watkins 2003) sowie die Lernkultur der Organisation am positivsten ausgeprägt sein sollten, wenn sowohl die Unterstützung durch die Führungskräfte $(=\mathrm{FO})$ als auch die SO hoch ausgeprägt sind. Vor diesem Hintergrund wird folgende Hypothese aufgestellt:

H1: Die höchsten Werte auf allen DLOQ-Skalen werden erreicht, wenn sowohl der Grad der SO als auch der Grad der FO hoch ist.

Auf Basis der genannten positiven Effekte der SDL ist anzunehmen, dass eine hohe Ausprägung der SO in Kombination mit niedriger FO bessere Ergebnisse in Bezug auf die Dimensionen des DLOQ bewirkt als vorrangig fremdorganisierte Weiterbildung. Demzufolge werden folgende Hypothesen aufgestellt:

H2: Die zweithöchsten Werte auf allen DLOQ-Skalen werden erreicht, wenn der Grad der SO hoch und der Grad der FO niedrig ist.

H3: Die dritthöchsten Werte auf allen DLOQ-Skalen werden erreicht, wenn der Grad der FO hoch und der Grad der SO niedrig ist.

Organisationen bzw. Führungskräfte, die Weiterbildung weder fremdorganisiert noch selbstorganisiert fördern, verfolgen einen Laissez-faire-Ansatz, d.h. sie überlassen Mitarbeiter/-innen sich selbst und adressieren Kompetenzentwicklungsfragen nicht explizit. Lowe, Kroeck und Sivasubramaniam (1996) weisen darauf hin, dass laissez-faire signifikant negativ mit Erfolgsindikatoren der Organisation zusammenhängt. Wenn Kompetenzentwicklung also weder von außen gefördert noch die Eigeninitiative des/der Mitarbeiters/-in unterstützt wird, ist folgender Zusammenhang anzunehmen:

H4: Die niedrigsten Werte auf allen DLOQ-Skalen werden erreicht, wenn sowohl der Grad der SO als auch der Grad der FO niedrig ist.

Ausgehend von der Unterscheidung der SO und FO von Lernen auf organisationaler Ebene stellt sich die Frage, welches Führungsverhalten aus der Sicht der Arbeitnehmer am vorteilhaftesten ist, um die Mitarbeiter/-innen bei der Kompetenzentwicklung zu unterstützen. In der Führungsforschung findet 
sich die Differenzierung zwischen aufgabenorientierter und mitarbeiterorientierter Führung (Rowold/Heinitz 2008), auf die im Folgenden zurückgegriffen wird. Auf den ersten Blick liegt es nahe, dass die mitarbeiterorientierte Führung für die Förderung der Kompetenzentwicklung der Organisationsmitglieder vorteilhafter ist. Bei dieser Art der Führung liegt der Fokus auf dem einzelnen Mitarbeiter/-in als Individuum und auf dessen/deren Bedürfnissen. Somit wird es für die Führungskraft möglich, individuelle Stärken und Potenziale der Geführten zu erkennen und diese gezielt zu fördern. Deswegen wird folgende Hypothese aufgestellt:

H5: Je mehr das Führungsverhalten dem Konzept der mitarbeiterorientierten Führung zuzuordnen ist, umso vorteilhafter ist es für die Kompetenzentwicklung der jeweiligen Mitarbeiter/-innen.

\section{Untersuchungsdesign}

Die Studie wurde in drei Pflegeeinrichtungen im Jahr 2011 durchgeführt. Es wurden insgesamt 110 Mitarbeiter/-innen (80\% Frauen) mittels eines standardisierten Fragebogens befragt. Die Auswertung erfolgte mit SPSS 20. SO von Lernen wurde mit fünf Items operationalisiert (Cronbach's Alpha = 0.71): 1 . Ihre Kompetenzen \& Kompetenzpotenziale müssen Mitarbeiter/-innen selbst einschätzen. 2. Jedes Organisationsmitglied entscheidet weitgehend autonom über seine Kompetenzentwicklung. 3. Neben der vom Unternehmen angebotenen Maßnahmen zur Kompetenzentwicklung können Mitarbeiter/-innen eigenständig Aktivitäten zur Kompetenzentwicklung auswählen, die dann auch vom Unternehmen unterstützt werden. 4. Organisationsmitglieder können autonom über ihre Kompetenzentwicklung und den Einsatz ihrer neu erworbenen Kompetenzen entscheiden. 5. Dafür, dass die Mitarbeiter/-innen ihre neu erworbenen Kompetenzen anwenden können, sind die Mitarbeiter/-innen selbst verantwortlich. „FO von Lernen” wurde mit sechs Items (Cronbach's Alpha $=0.73$ ) überprüft, die den FO-Index bildeten: 1 . Ob Organisationsmitglieder Kompetenzentwicklung betreiben, wird von jeder Führungskraft überprüft. 2. Es gibt Trainingseinheiten, die Führungskräfte dazu befähigen, entsprechende personalwirtschaftliche Instrumente einzusetzen. 3. Bei der Beurteilung von Führungskräften wird die Frage berücksichtigt, ob diese die Personalentwicklung ihrer Mitarbeiter/-innen systematisch unterstützen. 4. Kompetenzentwicklung wird als Möglichkeit der Leistungsverbesserung angesehen. 5. Ich habe die Möglichkeit der Verbesserung meiner Fähigkeiten in meinem Unternehmen. 6. Die Unternehmensleitung hat die aktuelle Unternehmensstrategie an seinen Mitarbeitern/-innen kommuniziert. Die weiteren acht verwendeten Skalen, die sich auf mehrere Items stützen, basieren auf Marsick 
und Watkins (2003) Ausführungen zu ihrem validiertem Fragebogen zur lernenden Organisation (DLOQ). Tabelle 1 gibt einen Überblick über Anzahl und Reliabilität der acht eingesetzten Watkins-Skalen. Zusätzlich wurde der Gesamtindex der Lernkultur gebildet, der von Marsick und Watkins zur Messung des allgemeinen Lernklimas vorgeschlagen wird.

Tab. 1: Skalenstatistiken für die DLOQ-Skalen

\begin{tabular}{|l|c|c|c|}
\hline Skala & $\begin{array}{c}\text { Anzahl der } \\
\text { Items }\end{array}$ & $\begin{array}{c}\text { Cronbachs } \\
\text { Alpha }\end{array}$ & Mittelwert \\
\hline Kontinuierliches Lernen & 7 & 0,79 & 3,66 \\
Rückfragen und Dialog & 5 & 0,82 & 3,71 \\
Zusammenarbeit/Lernen im Team & 4 & 0,82 & 3,18 \\
Methoden d. Erfassung von Lernen & 3 & 0,80 & 3,26 \\
Empowerment & 3 & 0,85 & 3,53 \\
Verbindungsstrukturen der Organis. & 3 & 0,84 & 3,87 \\
Strategische Führung & 4 & 0,87 & 3,95 \\
Wissensleistung & 3 & 0,86 & 3,82 \\
Lernkultur & 7 & 0,77 & 3,55 \\
\hline
\end{tabular}

Als Cut-Off-Wert für die Unterscheidung von niedriger und hoher FO bzw. SO wurde der Wert 3,0 (Antwort ,teils/teils”) festgelegt. Beschäftigte, deren Mittelwert auf der FO- bzw. SO-Skala zwischen 1,0 und 3,0 lag, wurden der Gruppe niedrige FO bzw. SO zugeteilt. Diejenigen mit einem Wert zwischen 3,01 bis 5,0 gehören dementsprechend zur Gruppe hohe FO bzw. SO. Für die Watkins-Skalen wurde jeweils der Skalenmittelwert zur Auswertung herangezogen.

Für die Auswertung der Mitarbeiter- bzw. Aufgabenorientierung im Hinblick auf das Führungsverhalten wurden je zwei Items verwendet. Die Mitarbeiterorientierung wurde durch folgende Items gemessen (Antwortmöglichkeit von 1 [nein] bis 5 [ja], Cronbachs Alpha 0,66): 1. Wird Ihre berufliche Weiterentwicklung durch Ihre Führungskraft gefördert? 2. Fühlen Sie sich in Ihrer Arbeit durch Ihre Führungskraft entsprechend gewürdigt? Die Aufgabenorientierung wurde ebenfalls mittels des Skalenmittelwerts von zwei Items erfasst (Cronbachs Alpha 0,72): 1. Mein Vorgesetzter zeigt mir, wie ich meine Leistung verbessern kann. 2. Mein Vorgesetzter sagt mir, was von mir erwartet wird.

\section{Ergebnisse}

91 Mitarbeiter/-innen haben die zugehörigen Fragen vollständig beantwortet und konnten einer der vier Gruppen der unterschiedl. Kombinationen von FO und SO zugeordnet werden: 1 . hohe $\mathrm{SO} /$ hohe $\mathrm{FO}, 36,3 \%$, 2. hohe $\mathrm{SO}$ /niedrige $\mathrm{FO}$, $13,2 \%$, 3. niedrige SO/hohe FO, 26, 3 \% und niedrige SO/niedrige FO, 24, $2 \%$. 
Zur Überprüfung der Hypothesen wurden die Skalen nach den vier Kombinationen von SO und FO ausgewertet (vgl. Tabelle 2). Es zeigt sich, dass die Gruppe der Befragten mit hoher SO und hoher FO auf fünf der acht Watkins-Skalen im Vergleich zu den anderen drei Gruppen den höchsten Skalenmittelwert hat (Bestätigung Hypothese 1). (Die Unterschiede bezogen auf die vier Gruppen erwiesen sich im Hinblick auf die acht Watkins-Skalen als signifikant.) Die Gruppe der Mitarbeiter/-innen mit hoher FO und niedriger SO hat auf drei der acht Watkins-Skalen den höchsten Skalenmittelwert. In Bezug auf den Gesamtindex der Lernkultur, der eine Aggregation der acht Skalen darstellt, haben beide Gruppen gleich hohe Skalenmittelwerte $(3,79)$.

Tab. 2: Gruppenzugehörigkeiten, Mittelwerte u. Standardabweichungen (SA) der vier Gruppen auf den DLOQ-Skalen (grau hinterlegt = Gruppe mit dem höchsten Skalenmittelwert)

\begin{tabular}{|c|c|c|c|c|}
\hline DLOQ-Skala & Gruppe & $\mathbf{N}$ & Mittelwert & $\mathbf{S A}$ \\
\hline \multirow{4}{*}{ Kontinuierliches Lernen } & Hohe FO, hohe SO & 31 & 3,25 & 0,63 \\
\hline & Hohe FO, niedrige SO & 18 & 3,37 & 0,55 \\
\hline & Niedrige FO, hohe SO & 12 & 2,76 & 0,84 \\
\hline & Niedrige FO, niedrige SO & 17 & 2,26 & 0,70 \\
\hline \multirow{4}{*}{ Rückfragen und Dialog } & Hohe FO, hohe SO & 31 & 3,74 & 0,96 \\
\hline & Hohe FO, niedrige SO & 24 & 4,02 & 0,92 \\
\hline & Niedrige FO, hohe SO & 12 & 3,33 & 1,08 \\
\hline & Niedrige FO, niedrige SO & 18 & 3,16 & 0,95 \\
\hline \multirow{4}{*}{$\begin{array}{l}\text { Zusammenarbeit und } \\
\text { Lernen im Team }\end{array}$} & Hohe FO, hohe SO & 33 & 3,43 & 0,78 \\
\hline & Hohe FO, niedrige SO & 21 & 3,48 & 0,77 \\
\hline & Niedrige FO, hohe SO & 12 & 2,83 & 1,26 \\
\hline & Niedrige FO, niedrige SO & 18 & 2,35 & 0,88 \\
\hline \multirow{4}{*}{$\begin{array}{l}\text { Methoden der Erfassung } \\
\text { von Lernen }\end{array}$} & Hohe FO, hohe SO & 32 & 3,59 & 0,82 \\
\hline & Hohe FO, niedrige SO & 20 & 3,38 & 0,67 \\
\hline & Niedrige FO, hohe SO & 11 & 3,18 & 1,43 \\
\hline & Niedrige FO, niedrige SO & 20 & 2,55 & 0,91 \\
\hline \multirow{4}{*}{ Empowerment } & Hohe FO, hohe SO & 31 & 3,89 & 1,12 \\
\hline & Hohe FO, niedrige SO & 19 & 3,46 & 0,62 \\
\hline & Niedrige FO, hohe SO & 12 & 3,08 & 1,18 \\
\hline & Niedrige FO, niedrige SO & 19 & 2,84 & 1,38 \\
\hline \multirow{4}{*}{$\begin{array}{l}\text { Verbindungsstrukturen } \\
\text { der Organisation }\end{array}$} & Hohe FO, hohe SO & 30 & 4,01 & 0,94 \\
\hline & Hohe FO, niedrige SO & 20 & 3,80 & 0,95 \\
\hline & Niedrige FO, hohe SO & 12 & 3,11 & 1,20 \\
\hline & Niedrige FO, niedrige SO & 17 & 3,14 & 1,07 \\
\hline \multirow{4}{*}{ Strategische Führung } & Hohe FO, hohe SO & 31 & 4,09 & 1,00 \\
\hline & Hohe FO, niedrige SO & 21 & 4,06 & 0,80 \\
\hline & Niedrige FO, hohe SO & 12 & 3,44 & 0,89 \\
\hline & Niedrige FO, niedrige SO & 17 & 2,81 & 0,79 \\
\hline \multirow{4}{*}{ Wissensleistung } & Hohe FO, hohe SO & 29 & 4,14 & 0,96 \\
\hline & Hohe FO, niedrige SO & 18 & 3,85 & 0,79 \\
\hline & Niedrige $\mathrm{FO}$, hohe SO & 11 & 3,48 & 1,13 \\
\hline & Niedrige FO, niedrige SO & 15 & 3,04 & 0,95 \\
\hline
\end{tabular}




\begin{tabular}{lllll}
\hline & Hohe FO, hohe SO & 28 & 3,79 & 0,69 \\
Gesamtindex: & Hohe FO, niedrige SO & 18 & 3,79 & 0,49 \\
Lernkultur & Niedrige FO, hohe SO & 11 & 3,16 & 1,11 \\
& Niedrige FO, niedrige SO & 14 & 2,87 & 0,54 \\
\hline
\end{tabular}

Die Gruppe der Befragten mit niedriger SO und niedriger FO erzielte auf sieben der acht Skalen und auf dem Gesamtindex der Lernkultur den im Vergleich niedrigsten Skalenmittelwert.

Ergänzend zur FO und SO des Lernens wurde in einem weiteren Schritt untersucht, ob das Führungsverhalten des Vorgesetzten mit den DLOQSkalen zusammenhängt (Korrelationsanalyse). Wie aus Tabelle 3 ersichtlich wird, ist die Korrelation zwischen der Mitarbeiterorientierung der Führungskraft und den DLOQ-Skalen deutlich höher als die Korrelation der entsprechenden Skalen mit der Aufgabenorientierung.

Tab. 3: Korrelation von Mitarbeiter- bzw. Aufgabenorientierung und den DLOQSkalen

\begin{tabular}{|c|c|c|c|c|c|c|c|c|c|}
\hline $\begin{array}{l}\text { Führungs- } \\
\text { ausrichtung }\end{array}$ & 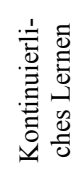 & 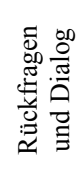 & 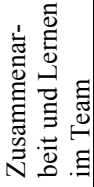 & 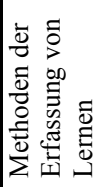 & $\begin{array}{l}\text { च् } \\
\text { : } \\
0 \\
0 \\
0 \\
\text { : } \\
\text { : }\end{array}$ & 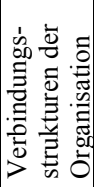 & 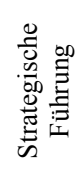 & 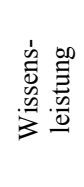 & 竞 \\
\hline Aufgaben- & 0,43 & 0,36 & 0,29 & 0,23 & 0,12 & 0,16 & 0,26 & 0,23 & 0,32 \\
\hline orientierung $\mathrm{N}$ & 82 & 90 & 87 & 85 & 84 & 82 & 83 & 76 & 73 \\
\hline Mitarbeiter- $\mathrm{R}$ & 0,52 & 0,50 & 0,47 & 0,33 & 0,26 & 0,33 & 0,47 & 0,28 & 0,49 \\
\hline orientierung $\mathrm{N}$ & 84 & 93 & 88 & 84 & 85 & 83 & 82 & 76 & 74 \\
\hline
\end{tabular}

\section{Diskussion}

\subsection{Grenzen der Untersuchung}

In zukünftigen Untersuchungen könnten die Items, die SO und FO abbilden, noch genauer bestimmt werden (weitere Differenzierung zwischen verschiedenen Ausprägungen von SO bzw. FO über die Einteilung in ,hoch” und „niedrig”). Die Differenzierung zwischen verschiedenen Ausprägungen der SO bzw. der FO könnte sich zudem in ausgeprägterer Form an inhaltlichen Kriterien orientieren.

Desweiteren wurde in der vorliegenden Studie bewusst auf die subjektive Einschätzung der Mitarbeiter/-innen zurückgegriffen, um die tatsächlich verhaltensbeeinflussende Wahrnehmung der Organisationsmitglieder zu erfassen. In zukünftigen Untersuchungen könnten diese Ergebnisse mit objektiven Messinstrumenten ergänzt und gespiegelt werden. Nicht zuletzt wurde im 
eingesetzten Fragebogen nur das Ausmaß abgefragt, in dem von Beschäftigten eine hohe SO des Lernens erwartet wird. Zukünftige Untersuchungen könnten darüber hinaus die Frage untersuchen, ob und in welchem Maße Beschäftigte diesen Anforderungen an die SO auch entsprechen, d.h. ob sie tatsächlich proaktiv der SO ihrer Lernprozesse nachgehen oder ob diese Anforderungen zwar bestehen, von den Organisationsmitgliedern aber nicht umgesetzt werden.

\subsection{Implikationen und wirtschaftspädagogische Relevanz}

Die vorliegende Studie untersucht den Zusammenhang zwischen FO, SO und Lernkultur. In der bisherigen Forschungsdiskussion wird primär auf die positive Rolle der SDL hingewiesen. Wie die Untersuchungsergebnisse zeigen, führt hohe SO jedoch nicht zwingend zu einer stark positiv ausgeprägten organisationalen Lernkultur. Eine Kombination aus hoher SO und hoher FO (Hypothese 1) war in der empirischen Studie mit besonders positiven Lernresultaten verbunden; ein Laissez-Faire-Ansatz (niedrige SO, niedrige FO; vgl. Hypothese 4) brachte, wie erwartet, die schlechtesten Ausprägungen auf den Lernkultur-Skalen mit sich. Die eingangs aufgestellten Hypothesen zur vergleichsweise positiven Verbindung von SO und Lernkultur bei niedriger FO (Hypothese 2) mussten jedoch verworfen werden. Anstatt dessen war an zweiter Stelle der positiven Ausprägung der Lernkultur die Kombination hohe FO, niedrige SO (Hypothese 3) zu finden. Somit scheint FO eine höhere Bedeutung für die Lernkultur eines Unternehmens zu haben als SO.

Diese Ergebnisse sind konträr zur bisherigen positiven Erwähnung der SDL. Eine mögliche Erklärung könnte darin gesehen werden, dass Mitarbeiter/-innen sich mit der SDL überfordert fühlen und nicht die notwendigen Kompetenzen besitzen, um Lernprozesse neben ihrer gewöhnlichen Arbeitstätigkeit proaktiv zu verfolgen. Eine weitere Erklärung könnte darin gesehen werden, dass der jeweilige Entwicklungsstand des Lernenden in Bezug auf selbstorganisiertes Lernen entscheidend ist. Den unterschiedlichen Fähigkeitsgrad im Hinblick auf die SDL gilt es bei künftigen Forschungsbemühungen noch stärker zu berücksichtigen.

Das Ergebnis, dass die FO von Lernen von hoher Bedeutung für die organisationale Lernkultur ist, führt zu der Frage, wie die Führungskräfte Lernen fremdorganisieren sollen. Die Ergebnisse zu Hypothese 5 weisen darauf hin, dass ein mitarbeiterorientierter Stil in höherem Maße mit der Lernkulturausprägung zusammenhängt als der aufgabenorientierte Stil. Ein weiterer Schritt könnte hier die weitere Ausdifferenzierung von Führungsstilen (z.B. transformational/transaktional) sowie die Erforschung dieses Zusammenhangs mit Kompetenzentwickung und Lernkultur sein. 
Als Implikation für die Wirtschaftspädagogik lässt sich schlussfolgern, dass Konzepte entwickelt werden müssen, welche die Kompetenzen zu selbstständigen Lernprozessen vermitteln, um die Verknüpfung zwischen institutionalisierten und selbstorganisierten Lernprozessen zu unterstützen. $\mathrm{Zu}-$ künftige Forschungsbemühungen sollten sich dabei auch damit beschäftigen, die Rolle der Selbststeuerungsdisposition zu untersuchen, welche sich auf interindividuell unterschiedliche Fähigkeiten und Bereitschaften zur Selbststeuerung von Lernen bezieht.

Für Mitarbeiter/-innen implizieren die Ergebnisse der vorliegenden Untersuchung, dass sie möglichst eine Führungskraft wählen sollten, die sie in der Kompetenzentwicklung unterstützt und in einer Coach-ähnlichen Rolle auch fremdorganisiert in die Kompetenzentwicklung eingreift. Falls diese Gegebenheit nicht vorhanden ist, könnte ein Arbeitsplatzwechsel und der damit verbundene Wechsel der Führungskraft eine mögliche Option sein. Das provokative Fazit in Bezug auf die Organisation der Kompetenzentwicklung kann demnach lauten: Führungskräfte sollten ihre Verantwortung in der Organisation in Hinblick auf die Organisation der Kompetenzentwicklung ihrer Mitarbeiter/-innen wahrnehmen und in einem dialogorientierten Prozess mit den Mitarbeitern/-innen gemeinsam die jeweiligen Stärken und Schwächen aufdecken, um die Stärken auszubauen und die Schwächen nicht zum Tragen zu bringen.

\section{Literatur}

Baethge, M/Baethge-Kinsky, V/Holm, R./Tullius, K. (2003): Anforderungen und Probleme beruflicher und betrieblicher Weiterbildung (Arbeitspapier 76). Düsseldorf: Hans-Böckler Stiftung.

Lowe, K.B./Kroeck, K.G./Sivasubramaniam, N. (1996): Effectiveness correlates of transformational and transactional leadership: A meta-analytic review of the MLQ literature. In: Leadership Quarterly, 7. Bd., H. 3, pp. 385-425.

Marsick, V. J./Watkins, K. E. (2003): Demonstrating the value of an organization's learning culture: The Dimensions of Learning Organizations Questionnaire. In: Advances in Developing Human Resources, 5. Bd., H. 2, pp. 132-151.

Park, S. (2008): Self-directed learning in the workplace. Online Submission, Paper presented at the Academy of Human Resource Development International Research Conference in the Americas (Panama City, FL, Feb 20-24, 2008).

Reckwitz, A. (2003): Grundelemente einer Theorie sozialer Praktiken. Eine sozialtheoretische Perspektive. Zeitschrift für Soziologie, 32(4), S. 282-301.

Rowold, J./Heinitz, K. (2008): Führungsstile als Stressbarrieren. In: Zeitschrift für Personalpsychologie, 7. Bd., H. 3, S. 129-140. 
Sembill, D./Seifried, J. (2006): Selbstorganisiertes Lernen als didaktische Lehr-LernKonzeption zur Verknüpfung von selbstgesteuertem und kooperativem Lernen. In: D. Euler, M. Lang/G. Pätzold: Selbstgesteuertes Lernen in der beruflichen Bildung. Stuttgart.: Franz Steiner, S. 93-108.

Voß, G. G./Pongratz, H. J. (1998): Der Arbeitskraftunternehmer. Eine neue Grundform der Ware Arbeitskraft? In: Kölner Zeitschrift für Soziologie und Sozialpsychologie, 50. Bd., H. 1, S. 131-158.

Watkins, K. E./Marsick, V. J. (2003): The value of a learning culture. In: Advances in Developing Human Resources, 5. Bd., H. 2, pp. 132-151.

Yang, B./Watkins, K. E./Marsick, V. J. (2004): The Construct of the Learning Organization: Dimensions, Measurement, and Validation. In: Human Resource Development Quarterly, 15. Bd., H. 1, pp. 31-55. 